\title{
An Integrated Neighbor Discovery and MAC Protocol for Ad Hoc Networks Using Directional Antennas *
}

\author{
Gentian Jakllari, Wenjie Luo and Srikanth V. Krishnamurthy \\ Department of Computer Science and Engineering, University of California, Riverside, \\ Riverside, CA, 92521 \\ \{jakllari,wluo,krish\}@cs.ucr.edu
}

\begin{abstract}
Many MAC sub-layer protocols for supporting the usage of directional antennae in ad hoc networks, have been proposed in literature. However, there remain two open issues that are yet to be resolved completely. First, in order to fully exploit the spatial diversity gains due to the use of directional antennae, it is essential to shift to the exclusive usage of directional antennae for the transmission and reception of all the upper layers frames. This would facilitate maximal spatial re-use and will efface the phenomena of asymmetry in gain. Second in the presence of mobility the MAC protocol should incorporate mechanisms by which a node can efficiently locate and track its neighbors.

In this paper we propose a new polling based MAC protocol that addresses both the issues in an integrated way. We perform analysis and extensive simulations to understand the performance of our scheme in terms of its ability to maintain connectivity, the achieved utilization efficiency, and throughput. We find that each node, on average, can achieve a per node utilization of about $80 \%$ in static and about $45 \%$ in mobile scenarios. Our protocol is seen to outperform both the traditional IEEE 802.11 MAC protocol and previosuly proposed protocols for use with directional antennae that provide partial solutions to solve the aforementioned problems. Finally, we also study the sensitivity of our protocol to various system parameters.
\end{abstract}

\section{Introduction}

Directional Antennae can help abate interference effects by either focusing the transmission energy in a particular direction or by tuning the antenna to receive the energy from a particular direction, or by doing both of the above [1]. The

*This work is supported in part by, the U. S. Army Research Office under the Multi-University Research Initiative (MURI) grant W911NF-04-10224, the NSF CAREER Grant 0237920 and the NSF NRT grant 0335302. benefits of using directional antennae in terms of achieving higher capacities, due to spatial diversity, have already been demonstrated in cellular networks via their deployment at base-stations [1]. Continuing reductions in the cost and size of antennae will soon make it feasible to use this technology in mobile terminals as well, especially as the operating frequency continues to increase. Hence, their use in ad hoc networks is foreseeable. As an example, in the $5.8 \mathrm{Ghz}$ ISM band, an 8-element cylindrical array will require a radius of only $3.3 \mathrm{~cm} \mathrm{[3].}$

Currently, there has been a lot of interest in terms of using directional antennae in ad hoc networks [5]-[10]. There have been papers that show that the spatial diversity due to the use of these antennae can provide significant gains in capacity [4]. In most of the work the use of directional antennae in static networks is considered [3], [6], [8], [12] There has been some limited work on dealing with mobility when directional antennae are deployed [3], [5], [10]. However, these schemes rely on omni-directional transmissions of control messages by nodes that try to reconnect with neighbors that move out of their angular range. Furthermore, most of the previously proposed schemes restrict themselves to either only directional transmissions or directional receptions. The inability of exclusively using directional antennae for both the transmission and reception of all MAC layer frames (control or data) results in two major problems: (a) the spatial re-use benefits are reduced due to the invocation of omni-directional communications and (b) the use of omni-directional receptions for certain packets and directional receptions for others leads to an inherent asymmetry in range. This phenomena can exacerbate the hidden terminal problem [8] and leads to a significant penalty in throughput.

A challenge associated with the exclusive deployment of directional antennae for all communications in mobile networks is that, due to the angular reduction in range in comparison to the omni-directional case, it is important for a node to poll each of its neighbors periodically to ensure that the neighbor's motion is tracked. The MAC protocols 
proposed thus far either completely ignore mobility or use omni-directional transmissions or receptions (thus inflicting the asymmetry in range problem) of HELLO messages to identify neighbors.

In this work, we propose a new MAC protocol for mobile ad hoc networks that addresses the issues mentioned above in an integrated way. We call our protocol PMAC for Polling-based MAC protocol. PMAC exclusively uses directional antennae for the transmission and reception of all the frames, i.e., we obviate omni-directional transmissions and receptions. Furthermore, the protocol facilitates the discovery of new neighbors by a node, and using polling, the maintenance of links to the discovered neighbors until they are outside the possible radial range of the node ${ }^{1}$. Polling is also used to schedule the transmissions and receptions of information. This would ensure that the transmitter and the receiver nodes point their antenna elements towards each other at the time that they are scheduled to communicate.

The rest of our paper is organized as follows. In Section II, we discuss prior related work on MAC protocols for use with directional antennae in ad hoc networks and state how our work differs from the prior schemes. We describe our polling based MAC protocol (PMAC) in detail in Section III. We perform a simple analysis to understand how quickly a node can discover its neighbors using our protocol and we present this analysis in Section IV. A layout of our simulation framework, the parameters used and the metrics that are of interest are provided in Section V. We present our simulation results and discuss these results in Section VI. Our concluding remarks form Section VII.

\section{RELATED WORK}

There has been a lot of interest in the design of MAC protocols for use with directional antennae in mobile ad hoc networks [5],[6],[7],[8],[10]. Efforts on routing and broadcasting using directional antennae in ad hoc networks are seen in [9],[13],[14]. In [15] a framework for a unified approach to the MAC design for the general case of smart antennae is presented.

Ko et al [6] propose a MAC protocol for use with directional antennae in static ad hoc networks. The scheme uses omni-directional transmissions/receptions of control messages. Furthermore, the experiments that the authors perform are based on the assumption that the sender knows the physical location of the receiver by means of GPS. Nasipuri et al [7] propose a MAC protocol for directional antennae based on carrier sensing. They examine the performance in simulations where speeds of up to $3 \mathrm{~m} / \mathrm{s}$ are considered. However, neither do they consider higher speeds nor do

\footnotetext{
${ }^{1}$ The radial range is the distance in the radial direction that a node can reach. In contrast the angular range specifies the angular beamwidth of the directional antenna being used.
}

they provide methods to track the mobility of users. Ramanathan [3] suggests that directional neighbor discovery be done by periodically sending HELLO messages. However, there is no discussion on the periodicity. Furthermore, without scheduled operations, HELLO messages may get lost and the ramifications are not studied. Takai et al [5] propose the use of directional virtual carrier sensing to access the channel. The scheme relies on angle of arrival or AOA caching to learn the destination's position; if no information is available in the cache, packets will be transmitted omni-directionally. If the mobility is high, the cached information becomes stale quickly (especially if the antenna beamwidth is small) and the protocol would have to frequently resort to omni-directional transmissions, the effects of which are not investigated. Choudhury et al [8] propose the multi-hop RTS protocol which is based on previous work in [6]. The authors identify that the radial range possible with directional transmissions combined with directional receptions is longer than that possible with directional transmissions combined with omni-directional receptions. Since the control packets (RTS and the CTS packets as in IEEE 802.11 ) are received omni-directionally, a multi-hop RTS is used to establish handshakes with distant neighbors that cannot be reached if those neighbors were to receive omni-directionally. Wang and Garcia-Luna-Aceves [12] discuss the interactions between spatial reuse and collision avoidance, and point out that omni-directional transmission of control packets may nullify the spatial reuse benefits.

Korakis et al [10] propose the use of a circular RTS (CRTS) message. By doing so, they achieve a higher range when transmitting the RTS message than with an omnidirectional transmission. The CRTS message can also help a node reconnect with neighbors that move out of its angular range. The CRTS scheme has been shown to outperform previously proposed MAC protocols. However, the scheme deploys omni-directional receptions which, in turn, can drastically reduce the possible increase in capacity. Our proposal to use fully directional communications eliminates the use of omni-directional transmissions. In spite of these previous efforts, as mentioned earlier, there are still two significant problems that arise with the deployment of directional antennae that remain unresolved.

1. Full exploitation of directional transmissions: Most of the solutions proposed, do not eliminate the requirement of omni-directional transmissions and/or receptions of control packets. This has three consequences. (a) it limits the frequency re-use significantly, (b) it limits network connectivity since the nodes are required to be within the omni-directional radius (fully directional communications can in some cases help bridge possible partitions that may arise with simply omni-directional communications) and (c) creates the 
problem of asymmetry in gain which in turn can decrease the network throughput[10].

\section{Locating and tracking neighbors under mobility:} In most of the previous work, the assumption that each node knows a neighbor's physical position so as to beamform correctly in the appropriate direction, is made. However, under mobility, the MAC protocol should offer a mechanism for a node to locate and track its neighbors. As mentioned, Korakis et al [10] use the CRTS approach to solve this problem. However, this approach suffers from a manifestation of the asymmetry in range problem.

To the best of our knowledge there is no MAC protocol that addresses both of these issues. The motivation to do so guides our design of our PMAC, described in Section III.

\section{The Polling-Based MAC Protocol}

In this section we describe our polling based medium access control protocol (PMAC) in detail. We use a scheme in which time is divided into contiguous frames. For our scheme, therefore, it is essential that each node in the network be synchronized with its neighbors in time. This requirement is not unreasonable since the nodes can synchronize during their polling slots (appropriate guard bands might be required). Furthermore, time-synchronization methods have been proposed for ad hoc networks [11]. If GPS is available it might be used to provide clock synchronization. It is possible that the nodes have different views of time as long as they are aware of the clocks of each of the neighbors that they communicate with.

The Frame Structure: The MAC protocol will allow a node to exist in one of three states;

- search state in which it searches for new neighbors

- polling state in which it polls known neighbors

- data transfer state wherein information is actually transferred.

As mentioned earlier, time is divided into contiguous frames as shown in Fig.1. Each frame is divided into three segments. The first segment is called the search segment. In each of the time-slots in the search segment, a node points its antenna (by appropriately adjusting its antenna weighting coefficients) in a randomly chosen direction. If communication is established with a new neighbor, messages are exchanged (the mechanism will be discussed later) and the two nodes agree to communicate on a regular basis in one of the slots in the polling segment, consistently, in subsequent frames.

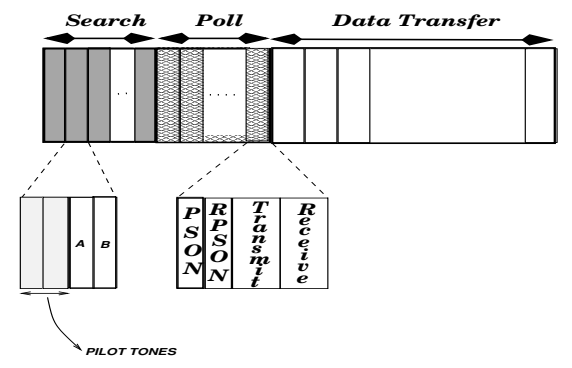

Figure 1. Frame Structure in PMAC

In the polling segment of each subsequent frame, the nodes schedule data transfers (in either direction) in the data segment. The communication in the particular polling slot takes place irrespective of whether the nodes have any data to exchange. Receiving this message from a neighbor helps a node adjust its antenna weighting coefficients for that neighbor, i.e., track the motion of the particular neighbor. The frame duration is a system parameter that should be appropriately chosen based on the expected mobility patterns. If nodes move with high speeds then the frame size should be small since, a node should poll its neighbors frequently in order to keep track of their positions. On the other hand, if there is little mobility, the frame size could be chosen to be longer. Clearly, in a dynamic network, where nodes move with different speeds, an optimal choice for the frame duration is difficult. If a neighbor moves out of the angular range of a node under consideration within a frame period, the node will have to rediscover the neighbor using the search slots. The trade-offs in terms of choosing a long versus short frame size will be discussed in Section VI.

The third part of the frame is used for scheduled data transfers.

Search Slots: In the search segment, each node searches for new neighbors. In each slot, the antenna is pointed in a randomly chosen direction. Each slot can be further divided to four sub-slots in each of which a particular sub-operation is performed. In the first sub-slot, the node would randomly choose to transmit its pilot tone (or identifier) or choose to receive. Both the transmissions and receptions are directional. If the node chose to receive in the first sub-slot it would transmit its pilot tone in the second sub-slot and vice versa. If there is a neighbor who has tuned his antenna in the same direction (in order to receive), when the node transmits, this neighbor will hear the pilot tone; it would correspondingly respond in second sub-slot. Sub-slots 3 and 4 are labelled sub-slot A and sub-slot B in Fig.1. In sub-slots $\mathrm{A}$ and $\mathrm{B}$, the nodes that successfully exchanged pilot tones, exchange $a$ list to specify the slots in their corresponding polling segments that are unused. The node that transmitted the pilot tone in the first sub-slot uses sub-slot A for transmitting its list; the other node of the pair transmits its list in 
sub-slot B. The two nodes with the help of each other's lists, then, identify a polling slot which can be used for scheduled polling. In our current implementation of PMAC, the pair of nodes pick the first common free polling slot for communicating on a periodic basis.

Polling Slots: The polling slots serve twofold: first, they allow two nodes to re-establish contact periodically so that they can track each other and ensure that the link is maintained. Second, they can be used in order to schedule data transfers in the third part of the frame. Once, the nodes agree upon a polling slot (as described earlier), they communicate in the same slot periodically frame after frame until they cannot communicate with each other due to their moving out of each other's radial range.

Each node announces, during its polling slot, the next data packet that it needs to send and its length. It also indicates the available instances in the data frame when it would be able to send or receive any data packet from the corresponding neighbor. If the node were to transmit in the first part of the polling slot, it first takes into account its own data packet's transmission before accounting for the neighbor's transfer in the data transfer portion of the frame. If on the other hand, the node was the recipient in the first part of the polling slot, then it accounts for the transfer of its neighbor's data before accounting for its own. Accordingly, each node schedules the announced data transmission/reception in the data transfer part of the frame. The data transfer is scheduled at the earliest time possible in either the current frame or in a future frame (in the corresponding data transfer segment) depending upon previously scheduled communications of each of the communicating nodes.

If the beamwidth is large or in some rare scenarios in which new neighbors move into the vicinity due to mobility, a collision can occur in a polling slot. In order to ensure that the collision is detected, we use a control message exchange as in the IEEE 802.11 MAC protocol. The PSON message (for Polling Slot ON) is similar to the RTS (Request to Send) message used in the IEEE 802.11 MAC protocol and the RPSON (for Response to Polling Slot ON message) is similar to the CTS (Clear to Send) message. In the event of two successive collisions (of any combination of the PSON and the RPSON messages), the two communicating nodes attempt to choose another polling slot. The nodes piggyback a list of their free polling slots at the end of each data exchange between them (in the data transfer part of the frame). Upon experiencing a collision, the nodes attempt to use the last common free slot (as per the list) in order to communicate with each other. If this were to fail they resort to a search. We alternate the order of transmission of polling messages. As an example, if a sender A transmits the PSON to a receiver $\mathrm{C}$ in frame $n$, then $\mathrm{C}$ would transmit the PSON to A in frame $(n+1)$. The node that sent the PSON, transmits in the first part (subslot A) of the polling slot and receives information in the second part (subslot B).

Data Transfer: The scheduled data transfers take place in the data transfer portion of the frame. If a given node has a data transfer scheduled with a neighbor at a particular time (in a previous polling slot), at that particular time, the node points its antenna in the direction of the neighbor. Similarly, the neighbor would have pointed its antenna in the direction of the node under discussion. The scheduled packets are then transferred from the sender to the receiver.

An RTS and CTS message are included prior to the data transfer in order to detect possible rare collisions. These collisions could occur either due to scheduling conflicts that arise due to large beamwidths or due to mobility effects (a new neighbor moves into the vicinity and has a conflicting communication scheduled in the same direction). These RTS and CTS messages are transmitted directionally. Note that the data packets could be of arbitrary length (in terms of a number of slots of some basic size); thus, packet transmissions are asynchronous within this part of the frame. We mean asynchronous in the sense that packet transmissions can begin at any basic slot boundary and the packet sizes are variable in terms of the number of basic slots. Typically the receipt of an RTS and CTS message of a different transmssion precludes a node from performing its own previously scheduled data transfer if it detects the possibility of causing interference. The node that detects a conflict simply refrains from sending its RTS or CTS message at the pre-scheduled time. The neighbor with whom the communication was scheduled infers that there has been a conflict in scheduling. The pair will then attempt to reschedule the data transfer in conflict at a later time, using the polling segment in the following frame. Note that the occurrence of such events are rare if the antenna beamwidth is sufficiently small.

Benefits of PMAC: Our schemes can either completely eliminate or alleviate the problems that are present in other previously proposed schemes.

Eliminating the problems due to range asymmetry: As mentioned earlier, since our protocol uses only directional transmissions it avoids the problem of asymmetry in gain. Furthermore the use of fully directional communications provides an increase in directional range (identified in [8]) that can benefit routing [9] in terms of computing shorter paths and bridging potential partitions that may exist when only omni-directional communications are used.

Handling Mobility: Previous schemes either completely ignore mobility or use omni-directional transmissions in order to detect neighbors that move out of angular range. In our scheme since we poll neighbors periodically, we ensure that each node is continuously aware of its neighbors' positions. Even in the presence of bursty traffic wherein a node may not exchange data with a neighbor for extended peri- 
ods in time, the polling of the neighbor helps the node track the neighbor.

Reducing the effects of deafness: Many of the previously proposed schemes suffer from the problem of deafness. The problem of deafness has been identified in [8]. When two nodes exchange control messages (RTS and CTS) directionally, a different neighbor of one of these communicating nodes may not hear the directional exchange. Later, during the data exchange between the nodes, this neighbor, being unaware of the data exchange might attempt to initiate communications with one of these nodes. However, clearly, it would not receive a response. This effect is referred to as deafness. As a consequence of the effects described, the neighbor would then back-off. The problem could repeat itself and may lead to the neighbor incorrectly concluding that a link failure has occurred. Since, our protocol is based on scheduled communications as opposed to asyncrhonous random access based communications, deafness does not occur.

Handling Multiple Types of Traffic: The data that can be exchanged can be of any type. Both real-time constant bit-rate traffic and bursty delay resistant traffic can be handled. If certain traffic types require QoS guarantees they can simply be scheduled before other queued delay-insensitive traffic.

\section{An Analytical Model to compute Neighbor Discovery time}

In this section, we develop a simple analysis to find the probability that, during the initialization of the network, a node takes $J$ frames to find and connect with a particular neighbor. We represent this probability by $P_{J}$. We also compute the probability that all of the node's neighbors are found within $J$ frames. To begin with, we make a set of assumptions and define certain parameters and metrics.

Assumptions:

- The network remains static for the duration of this preliminary search. This is reasonable since we expect the network initialization time to be fairly small as compared to the time it takes for the topology of the network to change drastically.

- The interference experienced by a receiver is limited to those interfering transceivers that are in its directional range and whose transmit beams are pointed toward that receiver. In such a case, where a receiver is the target for multiple such directional transmissions, we assume that a collision is experienced by the receiver.

- We begin counting frames from frame 1 upon initialization. Thus, the frame $i$ is the $i^{\text {th }}$ frame from network initialization.
- The notation "finding a neighbor", corresponds to first instance when the node discovers the neighbor ${ }^{2}$.

Parameters:

- The beamwidth of a directional antenna for either transmissions or receptions is fixed and is $\frac{2 \pi}{K}$, where $K$ is a system parameter. In other words, a node is capable of pointing its antenna in one of $K$ fixed directions.

- The node density, i.e., the number of nodes per unit area, $=\sigma$.

- The radial range of the antenna beam is $r$ units (in distance).

- The number of nodes that are within a transmit or receive beam is then $m=\frac{\pi r^{2}}{K} \sigma$. We assume for the ease of analysis, that for any node the number of neighbors within the node's transmit or receive beam is fixed and equal to $m$.

- The number of search slots per frame (a design parameter): $\eta$.

Metrics:

- $s$ : the probability that a node finds a particular given neighbor in a particular slot.

- $f$ : the probability that a node finds a particular given neighbor in a particular frame.

- $F_{i}$ : the probability that a node finds a particular neighbor in exactly the $i^{t h}$ frame.

- $P_{J}$ : the probability that a node discovers a particular neighbor in at most $J$ frames.

- $P_{m, J}:$ the probability that a node discovers all $m$ neighbors within an angular sector in at most $J$ frames.

- $P_{k, J}:$ the probability that $k$ neighbors are discovered within an angular sector in at most $J$ frames, where $k \leq m$.

In order for a particular node (say node A) to discover node $\mathrm{C}$ in a particular slot, its transceiver should be pointed towards C. The probability of this event is $\frac{1}{K}$. Similarly node $\mathrm{C}$ should point its antenna towards node $\mathrm{A}$. The probability of this event is $\frac{1}{K}$ as well. Furthermore, it is necessary that one of them should be in the transmit mode and the other should be in the receive mode. As mentioned earlier, a node could choose either the transmit or the receive mode

\footnotetext{
${ }^{2}$ Note that in subsequent search slots, the two nodes may synchronize their transmit and receive antennae; however, since they already have found each other, this is irrelevant.
} 
with probability $\frac{1}{2}$. None of the other $(m-1)$ nodes that can cause interference to the nodes' communication should be transmitting at the same time. Thus,

$$
s=2 \times \frac{1}{2 K} \frac{1}{2 K}\left(1-\frac{1}{2 K}\right)^{m-1}=\frac{1}{2 K^{2}}\left(1-\frac{1}{2 K}\right)^{m-1} .
$$

Correspondingly, the probability that $\mathrm{A}$ finds $\mathrm{C}$ in a particular frame that consists of $\eta$ search slots is:

$$
f=1-(1-s)^{\eta} .
$$

Accordingly, the probability that A finds $\mathrm{C}$ exactly in frame $i$ is given by:

$$
F_{i}=f(1-f)^{i-1} .
$$

Thus, the probability of $\mathrm{A}$ finding the node $\mathrm{C}$ in one of the first $J$ frames (since the event that $\mathrm{C}$ is found in frame $i$ is mutually exclusive from the event that $\mathrm{C}$ is found in frame $k$ for $i \leq J$ and $k \leq J$ and $i \neq k$ )

$$
P_{J}=\sum_{i=1}^{J} F_{i}=\sum_{i=1}^{J} f(1-f)^{i-1} .
$$

Simplifying this expression, we get

$$
P_{J}=1-\left(1-\frac{1}{2 K^{2}}\left(1-\frac{1}{2 K}\right)^{m-1}\right)^{\eta J} .
$$

Since the event of finding a particular neighbor is independent of the event of finding another particular neighbor ${ }^{3}$, we compute the probability of finding $k$ neighbors within the particular angular sector in $J$ frames to be:

$$
P_{k, J}=\left(\begin{array}{c}
m \\
k
\end{array}\right) P_{J}^{k}\left(1-P_{J}\right)^{m-k} .
$$

Then, the possibility of finding all $m$ neighbors within the angular sector in $J$ frames is given by:

$$
P_{m, J}=\left(1-\left(1-\frac{1}{2 K^{2}}\left(1-\frac{1}{2 K}\right)^{m-1}\right)^{\eta J}\right)^{m} .
$$

We plot the probability of finding all directional neighbors (within an angular sector) versus the antenna beamwidth for an example topology in Fig.2. The topology considered consists of 12 nodes in total, placed uniformly around the node that we're interested in. Thus, for different antenna beamwidths, $m$ varies. For this experiment, we fix the number of search slots in the frame (SSL) to be 20 .

From Fig.2, we see that for the assumed simple topology, the larger the antenna beam, the higher the probability that a particular neighbor will be found within a specified number of frames $(J)$. This result is expected since the larger

\footnotetext{
${ }^{3}$ Note that a node that is already discovered still contributes to interference effects. Thus, the interference effects experienced and the possibility of collision is the same throughout. This is the reason why the discovery of a node is independent of the discovery of any other node.
}

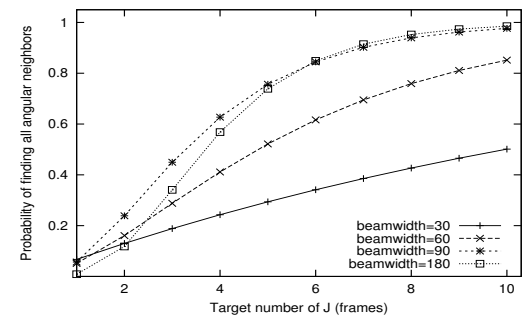

\section{Figure 2. Probability of finding all angular neighbors within $J$ frames}

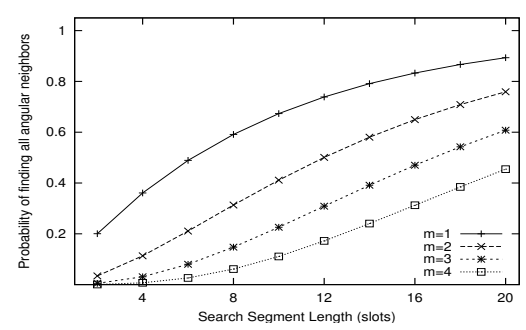

Figure 3. Probability of finding all angular neighbors within 8 frames with different number of search slots in one frame

beam corresponds to fewer angular segments, which leads to a greater likelihood of neighbors aligning their antennae towards each other.

Next, we study the neighbor discovery efficiency for various node densities. An antenna beamwidth of $60^{\circ}(K=6)$ is assumed. The node density is varied such that $m$ varies between 1 and 4 .

The probability of a node finding all of its neighbors in a particular sector within a period of 8 frames is plotted versus the size of the search segment in Fig.3. Clearly, as one might expect, with an increased value for $m$ (higher number of neighbors) it takes a longer time to discover all of the neighbors. Even with 3 nodes per sector (a node degree of 18), one finds that if the size of the search part of the frame is 20 slots, the probability that all of the neighbors within a particular sector can be found in 8 frames is 0.6 . If the degree of a node is moderate (from 6 to 8 ) we see that this probability could be as high as 0.9 .

\section{Simulation Model and Framework}

Our simulations are performed in Opnet, version 10.0 [16]. We chose Opnet because it offers the antenna editors which allows the creation of arbitrary antenna gain beams in 3-D. After creating a directional antenna, Opnet also provides system calls for pointing the main beam of 


\begin{tabular}{|l|l|}
\hline Search Segment Length (SSL) & 20 (slots) \\
\hline Poll Segment Length (PSL) & 4 (slots) \\
\hline Data Transfer Segment Length (DTSL) & 800 (slots) \\
\hline Packet size in slots 1,2 in Search Segment & 10 bytes \\
\hline Packet size in slots A,B in Search Segment & 20 bytes \\
\hline Poll packet size & 20 bytes \\
\hline Poll reply packet size & 14 bytes \\
\hline PSON packet size & 20 bytes \\
\hline RPSON packet size & 14 bytes \\
\hline RTS packet size & 20 bytes \\
\hline CTS packet size & 14 bytes \\
\hline Data packet size & 512 bytes \\
\hline Frame size & 1.64 seconds \\
\hline Data Transmission Rate & 2 Mbps \\
\hline Channel frequency & $2.4 \mathrm{Ghz}$ \\
\hline Antenna gain & $20 \mathrm{db}$ \\
\hline &
\end{tabular}

Table 1. Simulation Parameters

the directional antenna at an arbitrary point in 2-D space. We place nodes in a two dimensional flat terrain area. We either choose specific topologies (as will be specified) or place nodes randomly. Each node generates CBR. When a packet is generated, its destination is randomly chosen to be one of the node's neighbors. In our mobility model each node randomly chooses a location in the terrain and moves towards this destination with fixed speed. Upon arriving at the destination, a new location is chosen and the procedure is repeated. We choose a speed of $2 \mathrm{~m} / \mathrm{s}$ to represent a pedestrian environment and a speed of $10 \mathrm{~m} / \mathrm{s}$ to represent vehicular environments.

We denote the number of search slots in a frame by Search Segment Length (SSL), the number of poll slots by Poll Segment Length (PSL), and the number of slots in the data transfer segment (each slot is equal in size to that of a data packet) by Data Transfer Segment Length (DTSL). We use the system parameters listed in Table I unless specified otherwise. In each polling slot we limit the maximum channel time that can be reserved in the data transfer part to at most the time taken to transmit 400 packets (each of 512 bytes). We impose this restriction since we do not want a single node to dominate channel access. Note that reservations can be made for data transfers in subsequent frames. We also require that reservations be made for at most four frames in advance. This restriction is necessary since, in conditions of mobility, if a node is allowed to make reservations in the too distant future, it may actually move out of range by the time that the reservation is honored.

We are interested in two performance metrics:

- Total Network Throughput: The number of packets successfully transported at the MAC layer per unit time. Since PMAC incorporates the function of neighbor discovery upon initialization, we take care to exclude the initial time duration in our simulations to account for this phase of operations ${ }^{4}$.

- Per node Channel Utilization Ratio (CUR): The fraction of time that a node either transmits or receives useful information over the total simulation time.

\footnotetext{
${ }^{4}$ This initial duration was observed to be approximately 2 seconds on average.
}

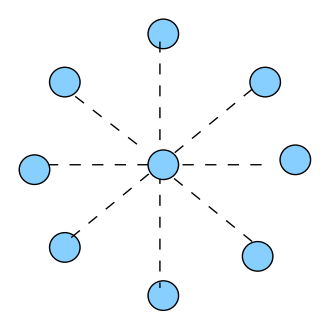

Figure 4. Star topology for the evaluation of the neighbor discovery process.

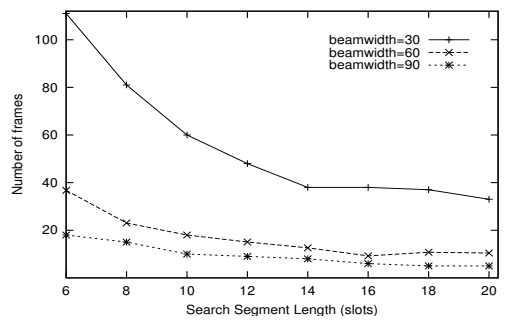

Figure 5. Number of frames required for center node to find all possible neighbors in star topology

\section{Simulation Results and Discussions}

In this section, we evaluate the performance of PMAC in terms of the defined metrics. We also study the sensitivity of the performance to various system parameters. Each of our simulations is run for 500 seconds. In each graph, every point is an average computation over 20 simulation runs.

The Neighbor Discovery Process: In our first experiment we consider the star topology shown in Fig.4 to study PMAC in terms of the efficiency in discovering neighbors at initialization. We had a simple analytical model in a previous section to compute certain probabilistic metrics that quantify the efficiency of the neighbor discovery process. Our simulation experiments with the realistic channel models provide an estimate of the time duration taken by the node at the center of the star topology to discover all of its neighbors. We vary the number of slots in the search segment as well as the antenna beamwidth and compute the number of frames required by the node in order to discover its neighbors.

As shown in Fig.5, with an increase in SSL, the number of frames that is required in order for the node to discover all of its neighbors decreases. When $S S L=20$, the node at the center takes, on average, 5 frames to find all of its neighbors by using a $90^{\circ}$ antenna beam. If the antenna beam is $60^{\circ}$, the node takes about 10.5 frame durations on average. 
Clearly, the smaller the beamwidth, the higher the delay incurred in the initial discovery process. As an example, with a $30^{\circ}$ antenna beam, when $S S L=6$, the required number of frames is more than 100 . Note that with a 1.64 second frame size, this translates to less than 3 minutes. This might be acceptable since this search process is only required during the initialization phase. Furthermore, note that in order to ensure strong connectivity, a node does not need to discover all of its neighbors. Data transfer can in fact be performed to a neighbor that is not discovered via a multi-hop route from one of the neighbors that is already discovered. We examine this in more detail in our next set of experiments.

Required Number of Polling Slots: In each polling slot, a node can poll exactly one neighbor. In other words, given a fixed Poll Segment Length (PSL) $p$, a node can talk to at most $p$ of its neighbors. In reality, however, due to scheduling conflicts, the number of neighbors that a node can communicate with may be less than $p$. Clearly however, the number of nodes that a node can communicate with ( say $\alpha$ ) increases if we increase the PSL. On the other hand, this would increase the protocol overhead, and thus, could cause a reduction in the maximum achievable channel utilization. We re-iterate here that the frame size is chosen based on the mobility in the network. As mentioned earlier, a node must poll each of the neighbors with which it has established connections every so often in order to track the mobility of these neighbors. The frame time is chosen such that this is feasible. If one were to increase the number of polling slots while this frame size is held constant because of the above constraint, one would see a decrease in the utilization efficiency. Therefore, it is important to choose a certain sub-set (say $\beta$ ) of the $\alpha$ neighbors $(\beta<\alpha$ ), such that the other $\alpha-\beta$ neighbors are reachable via the $\beta$ chosen neighbors via multi-hop paths. In other words, we need to ensure that there are no network partitions. We need to choose an appropriate value of PSL, such that, most of the nodes in the network can establish the connections either via a single hop or via multiple hops to all the other nodes in the network.

To efficiently evaluate the possibility of network partitions for a chosen value of PSL, we introduce the concept of network reachability $\gamma$. This is defined to be the ratio of the number of other nodes that a node can actually reach to the total number of nodes that it could potentially reach. For a strongly connected network with $n$ nodes, the total number of nodes that a node could potentially reach is $n-1$.

However, if the node could only reach $x$ of the other $n-1$ nodes $(x \leq n-1)$ for a given value of PSL, the network reachability for that node is given by: $\gamma=\frac{x}{(n-1)}$.

In order to study the effect of varying the PSL, we consider two scenarios. First, we consider a hexagonal network with 44 nodes: each node has 6 one-hop neighbors except

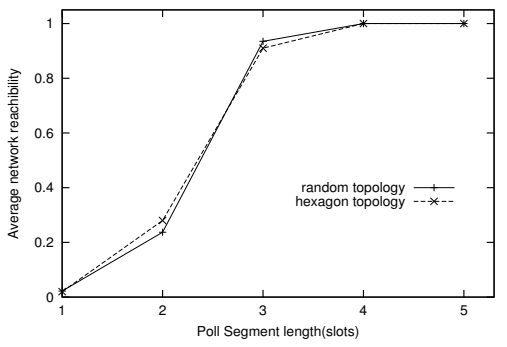

\section{Figure 6. Network Reachability test for ran- dom and hexagon topology with $30^{\circ}$ antenna}

for the border nodes. Second, we consider a randomly generated topology with 40 nodes in a $1000 \mathrm{~m} \times 1000 \mathrm{~m}$ flat terrain region. We vary the size of PSL, and for each value, we compute the average network reachability. The simulation results for the two topologies are shown in Fig.6.

The chosen antenna beamwidth for the experiments (for the results plotted in Fig.6) is $30^{\circ}{ }^{5}$. As one might expect, the average network reachability is low when a node's PSL is just 1 or 2 slots $^{6}$. Note however, that even with as few as 3 slots per frame the network is more than $90 \%$ connected. These results show that a very small number of polling slots (3 to 4) are sufficient to ensure almost complete network reachability at moderate node densities.

Performance in terms of throughput, latency, PDR and CUR: Next, we investigate the performance of our protocol performance in terms of the metrics defined in Section V: total network throughput, the per node packet delivery ratio $(P D R)$, and the per node channel utilization ratio $(C U R)$.

First, we investigate the performance of our protocol in terms of throughput. We compare the performance with that of the IEEE 802.11 MAC and the CRTS scheme proposed in [10]. The reason we choose CRTS is that, as with PMAC, it integrates the node discovery process as a part of the MAC layer. Furthermore, it has been shown in [10] that the scheme outperforms prior proposed schemes. We choose 8 antenna beams ${ }^{7}$. We run our simulations using two topologies. In the first one we place 15 nodes randomly in the region of interest. Each node is mobile with a velocity $10 \mathrm{~m} / \mathrm{s}$. The results are depicted in Fig.8. As seen, both PMAC and CRTS provide significant improvements over the IEEE 802.11 performance as one might expect. How-

\footnotetext{
${ }^{5}$ Experiments were done for antenna beamwidths $60^{\circ}$ and $90^{\circ}$ but are not presented since the general behavior was found to be the same for those cases as well.

${ }^{6}$ Note that even if the node has a large SSL, the number of neighbors that it can communicate with depends on the PSL.

${ }^{7}$ This seemed to be reasonable in terms of achieving a good level of spatial re-use. With a larger number of beams (each of which is narrower), the overhead with the CRTS scheme further increases.
} 
ever note that PMAC outperforms CTRS. This is due to the fact that CTRS requires control overhead per data packet transmission (due to circular RTS messages) and this increases with the number of antenna elements. With PMAC this overhead is independent of the number of antenna elements.

For the second experiment we choose a grid topology of 16 nodes. The results are depicted in Fig.7. In this case as the load increases so does the difference between the performance of PMAC and CRTS. We find that under heavy load the later suffers by the asymmetry in gain due to omnireception of the RTS/CTS packets from the neighbors of transmitter and receiver. In [10] the authors use the circular RTS to address this issue. By doing so they notify all the transmitter's neighbors of the intended communication, therefore the data packet transmission is protected. However since the CTS is transmitted only toward the transmitter the receiver's neighbors are not notified and therefore, the ACK is exposed to the asymmetry. Although the ACK duration is small, under heavy load, in the grid topology ACK collisions create significant problems. On the other hand, PMAC, using only scheduled directional communications does not suffer from these problems.

Next we place twenty five nodes, uniformly distributed in a $500 \mathrm{~m} \times 500 \mathrm{~m}$ flat terrain; each of these nodes generates CBR traffic with a packet size of 512 bytes. There is no mobility unless otherwise specified. Fig.9 displays the per node channel utilization ratio. As we see the channel utilization ratio increases with load. The maximum CUR achieved is about $78 \%$ with a $30^{\circ}$ antenna beamwidth. Under ideal conditions, one can compute the maximum achievable CUR (an upper bound) to be:

$$
C U R_{\max }=\frac{\text { data slot size } \times \text { DTSL }}{\text { frame size }},
$$

where DTSL is the Data Transfer Segment Length (defined in section V). This bound is computed with the assumption that the data transfer portion of the frame is completely utilized. With the parameters that we use, this turns out to be $97.56 \%$.

There are several factors that contribute to the degradation of the actual CUR in realistic scenarios: (a) The wastage of channel bandwidth during the initialization phase wherein the node finds its neighbors. (b) The scheduling is done in the order in which nodes are polled and hence, might lead to a sub-optimal utilization of the data part in the frame. (c) Possible collisions of PSON and RPSON messages and to a lesser extent the RTS and CTS messages. (d) the topology of the network may not facilitate complete utilization. As an example, in the star topology in Fig.4, the CUR that is achievable by the nodes around the center node may be expected to be $\frac{1}{8} \times 100=12.5 \%$. On the other hand, the center node may see a $100 \%$ CUR. Nevertheless

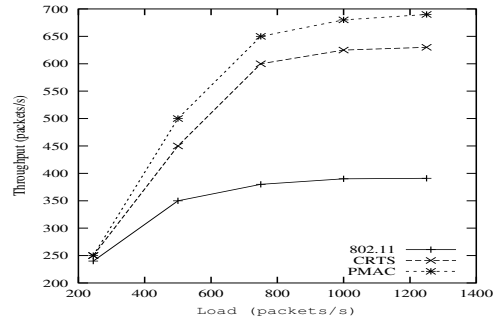

Figure 7. Total Network Throughput vs. Network Traffic Load in mobile random topology

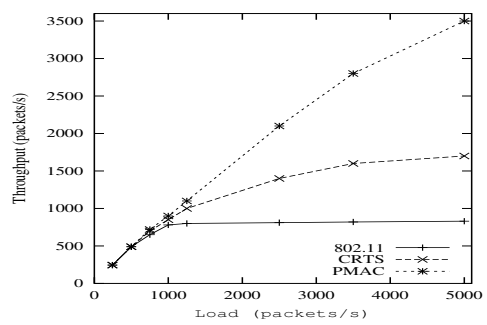

Figure 8. Total Network Throughput vs. Network Traffic Load in stationary grid topology

we point out that the CUR of $78 \%$ achieved by PMAC is fairly high for ad hoc networks.

Effects of Mobility: Fig.10 and Fig.11 show the CUR for the cases wherein the nodes are now mobile. In the network considered in Fig.10 nodes move with a speed of 2 $\mathrm{m} / \mathrm{s}$ and in the network considered in Fig. 11 the nodes move with a speed of $10 \mathrm{~m} / \mathrm{s}$. In these experiments, if a node generates packets for a neighborhood node that it is unable to communicate with any more (due to the node moving out of range), it simply discards those packets. We observe from Fig.10, the channel utilization degrades as compared with the stationary case by as much as $40 \%$. In mobile scenarios, transient topological effects (as described earlier) can cause a degradation in the CUR. An additional factor that comes into play is the possibility of an increased number of scheduling conflicts in both the polling part and the data part that cause collisions as nodes move around. The former results in a need for the rediscovery of neighbors using the search part. Reservations made for that neighbor are wasted. These factors result in an additional wastage of channel capacity. Another interesting factor to note is that mobility immediately causes a degradation in performance; the difference in the performance of the protocol with speeds of $10 \mathrm{~m} / \mathrm{s}$ and $2 \mathrm{~m} / \mathrm{s}$ does not seem to be significant for the chosen frame size. We wish to point out that even with this degradation, each node in the network (even at speeds of $10 \mathrm{~m} / \mathrm{s}$ ) is able to use the channel efficiently 


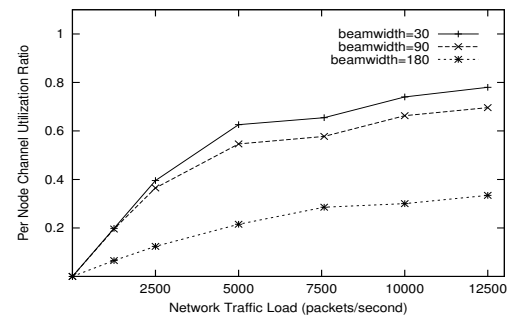

Figure 9. Per Node Channel Utilization Ratio
vs. Network Traffic Load in stationary random
topology

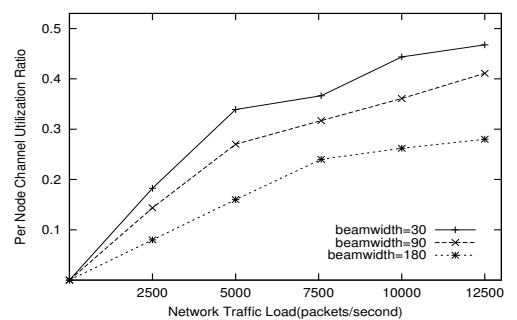

Figure 10. Per Node CUR vs. Network Traffic Load in random topology with speed $=2 \mathrm{~m} / \mathrm{s}$

for transport of useful information about $40 \%$ of the time. Thus, in total, all the nodes together attribute to a much higher channel utilization in the network.

As described earlier, in order to ensure that a node is tracked while it moves, the frame size is to be chosen appropriately. If the frame size is too long, nodes would frequently move out of range. If on the other hand, the frame size too small we incur a large overhead. Our goal is to examine the sensitivity of the maximum achievable CUR to variations in frame size. Towards this, we set the offered traffic load to 12,500 packets/ second (which is the maximum load that the network can handle with the $2 \mathrm{Mbps}$ data rate.) and vary the frame size (vary the number of data slots per frame). The number of polling slots and search slots are kept fixed at 4 and 20 respectively as before ${ }^{8}$.

Fig.12 and Fig.13 show the maximum CUR versus the frame duration, with moving speeds of $2 \mathrm{~m} / \mathrm{s}$ and $10 \mathrm{~m} / \mathrm{s}$, respectively. Note that we observe the expected behavior in our experiments. For small frame sizes the CUR suffers because of excessive overhead. As we increase the frame

\footnotetext{
${ }^{8}$ Note that one could potentially convert the search slots to polling slots or in fact, reduce the overhead per frame by eliminating some of the search slots after the initialization process. However, this would need synchronization among nodes and since, then, the duration of the search for different nodes is different we do not do this. Providing such modifications for enhancing the efficiency of the scheme are beyond the scope of this paper and will be studied in future work.
}

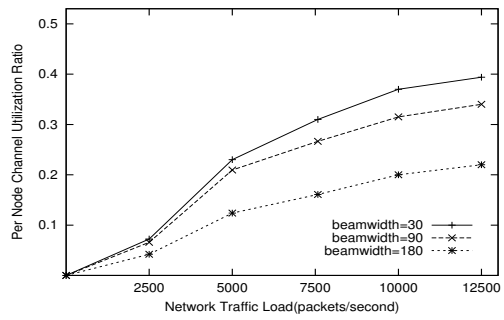

Figure 11. Per Node CUR vs. Network Traffic Load in random topology with speed $=10 \mathrm{~m} / \mathrm{s}$

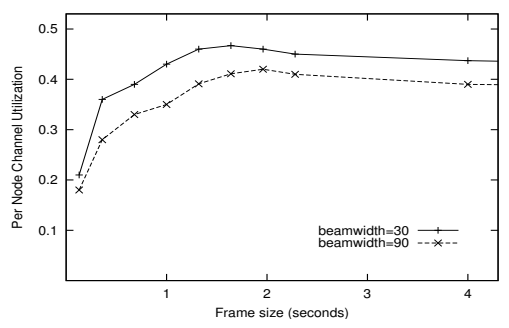

Figure 12. Per Node CUR achieved vs. Frame size in random mobile topology with speed = $2 \mathrm{~m} / \mathrm{s}$

size we see an increase in the CUR. However, for larger values the CUR in fact begins to drop. The drop is more prominent at higher speeds. With an increased beamwidth, one might expect that the polling interval can be potentially longer since nodes stay within the angular range of a node for longer periods in time. This effect is visible in Fig.12 and Fig.13, i.e., with a $90^{\circ}$ beamwidth, the maximum CUR occurs at a frame size that is larger than the frame size at which the maximum CUR occurs if the beamwidth is $30^{\circ}$. Note that the per node maximum channel utilization drops with increased beamwidth due to higher interference effects. Furthermore, the maximum CUR achieved drops as we increase the speed (from $2 \mathrm{~m} / \mathrm{s}$ to $10 \mathrm{~m} / \mathrm{s}$ ) since, now, we would need a smaller frame size and hence incur higher overhead. We reiterate that even with this, as shown, PMAC outperforms the previously proposed CRTS and the IEEE 802.11 schemes especially for small antenna beamwidths and in regular structured topologies.

\section{Conclusions}

In this paper, we propose a MAC protocol for use with directional antennae in mobile ad hoc networks. Our protocol overcomes the problems due to asymmetry in range and deafness when these antennae are deployed. Furthermore, it efficiently handles mobile scenarios by facilitating the dis- 


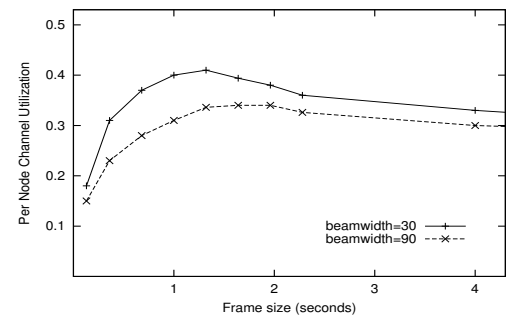

Figure 13. Per Node CUR achieved vs. Frame size in random mobile topology with speed = $10 \mathrm{~m} / \mathrm{s}$

covery of new neighbors by a node and the maintenance of links to the discovered neighbors. The key idea that forms the basis for our protocol is the use of a polling strategy wherein a node polls its discovered neighbors periodically; this would enable the node adjust its antenna weighting coefficients so as to continuously track its neighbors.

We perform extensive simulations to evaluate our protocol and we find that we achieve an extremely high per node channel utilization of up to $80 \%$ in static scenarios and up to $50 \%$ in mobile scenarios. Our protocol outperforms the IEEE 802.11 MAC and the previously proposed CRTS scheme that has been shown to outperform most other MAC protocols for use with such antennae. We also provide a simple analytical model to estimate the efficiency of the mechanism that we use for neighbor discovery; we compute the probability distribution of the duration of the search process. Our results seem to indicate that PMAC is a viable option for use with directional antennae in ad hoc networks and can provide high capacities even in highly mobile scenarios.

\section{References}

[1] J.C.Liberti Jr. and T.S.Rappaport, Smart Antennas for Wireless Communications: IS95 and Third Generation CDMA Applications, Prentice Hall, April 1999.

[2] S.V.Krishnamurthy et al, "Polling-Based Media Access Protocols for use with Smart Adaptive Array Antennas", in IEEE/ACM Transactions on Networking, April 2001.

[3] R.Ramanathan, "On the Performance of Ad Hoc Networks with Beamforming Antenanas", in Proceedings of ACM MOBIHOC, 2001.

[4] S.Yi et al, " On the Capacity Improvement of $\mathrm{Ad}$ Hoc Wireless Networks using Directional Antennas", in Proceedings of ACM MOBIHOC, 2003.
[5] M.Takai et al, "Directional Virtual Carrier Sensing for Directional Antennas in Mobile Ad Hoc Networks", in Proceedings of ACM MOBIHOC, 2002.

[6] Y.Ko et al, "Medium Access Control Protocols Using Directional Antennas in Ad Hoc Networks", in Proceedings of IEEE INFOCOM, 2000.

[7] A.Nasirpuri et al, "A MAC Protocol for Mobile Ad Hoc Networks Using Directional Antennas", in Proceedings of IEEE WCNC, 2000.

[8] R.Roychoudhury et al, " Using Directional Antennas for Medium Access Control in Ad Hoc Networks", in Proceedings of ACM MOBICOM, 2002.

[9] R.Roychoudhury and N.H.Vaidya, "Impact of Directional Antennas on Ad Hoc Routing", in Proceedings of IFIP Personal and Wireless Communications (PWC), 2003.

[10] T.Korakis et al, “ A MAC Protocol for Full Exploitation of Directional Antennas in Ad-hoc Wireless Networks", in Proceedings of ACM MOBIHOC, 2003.

[11] K.Romer, "Time Synchronization in Ad Hoc Networks", in Proceedings of ACM MOBIHOC, 2001.

[12] Y.Wang and J.J.Garcia-Luna-Aceves, "Spatial Reuse and Collision Avoidance in Ad Hoc Networks with Directional Antennas", in Proceedings of IEEE GLOBECOM, 2002.

[13] S.Bandopadhyay et al, " A Network-Aware MAC and Routing Protocol for Effective Load Balancing in Ad Hoc Wireless Networks with Directional Antennas", in Proceedings of ACM MOBIHOC, 2003.

[14] A.Spyropoulos and C.S.Raghavendra, "Energy Efficient Communications in Ad Hoc Networks Using Directional Antennas", in Proceedings of IEEE INFOCOM, 2002.

[15] K. Sundaresan and R. Sivakumar, "A Unified MAC Layer Framework for Ad-hoc Networks with Smart Antennas", in Proceedings of ACM Mobihoc, 2004

[16] Opnet User's Documentation, http://www.opnet.com. 